\title{
The effect of familiarization instructions on associative latency and learning
}

This experiment investigates the effects of using different familiarization instructions (Spell or Pronounce) on associative latency and learning. Significant effects (reduction in associative latency and the number of errors to criterion) were obtained only with instructions to pronounce. The implications of this phenomenon are discussed in terms of the effect of familiarization on meaningfulness. The reasons for discarding present, widely-used definitions of meaningfulness are cited.

Recent evidence supports the conclusion that familiarization reduces the latency of Ss' responses to CVC trigrams (Gillooly, 1965) in spite of the earlier data (Riley \& Phillips, 1959). Further, it was suggested that it is the familiarization instructions given Ss (Pronounce or Spell) that may be the determinant of the effect. However, the reported experiments (1965) were not designed to test such an hypothesis with the result that there was some confounding of variables-response mode was not the only part of the experimental task that was allowed to vary.

This study seeks to determine (1) if the reduction in associative latency (AL) can be replicated with different Ss and materials, (2) if the familiarization instructions (specifically, mode of response) are the determinant of the effect, and (3) if the reduction in $\mathrm{AL}$ is associated with facilitation in a learning task.

Based on the results of the previous studies, it is hypothesized that with respect to AL: (abbreviated terms explained in Procedures, and > means "is significantly faster than" ") $\mathrm{H}_{1}$ : PRF $>$ PIF, $\mathrm{H}_{2}$ : SRF $>\mathrm{SIF}$, therefore, $\mathrm{H}_{3}$ : $\mathrm{PRF}>\mathrm{SRF}$. With respect to the learning data, it is hypothesized that there will be facilitation wherever AL is reduced in addition to whatever other facilitation is observed. It should be noted that these hypotheses call for the use of one-tailed tests of significance.

\section{Method}

Experimental Design: The design is a 2 by 2 factorial with two sets of instructions (Spell/Pronounce) and two kinds of familiarization (Relevant/Irrelevant). The dependent variables a re associative latency and, in the learning task, the number of errors to criterion.

Subjects: Forty-four male undergraduate students who were enrolled in an introductory psychology course were randomly assigned to the four conditions. The use of anova was planned, however, circumstances prevented its use.

Materials and Apparatus: Two lists of 12 CVC trigrams were constructed from the lists in Hilgard (1951). List 1 was column 6 (22.7 average association value according to Glaze, 1928) in Hilgard's Table 7 (p. 540) and list 2 was composed of orthographically different items (no item in list 1 and 2 shared any two letters in common) of low association value from the same table. Associative latencies were measured with a Standard electric timer calibrated in $.01 \mathrm{sec}$. and activated by telegraph key. The trigram lists were pre- sented in the serial anticipation task by a Gerbrands memory drum.

Procedure: The experiment consisted of three parts-familiarization (on both list 1 and 2), immediately followed by an association test (on list 1 stimuli only), which, in turn, was immediately followed by a serial anticipation task (on list 1 only). The only parts of the procedure which varied were the familiarization instructions and the materials. Familiarization on list 1 is "Relevant Familiarization" (RF) and familiarization on list 2 is "Irrelevant Familiarization" (IF).

Familiarization: In individual sessions, Ss were given a pack of 84 3 in. $x$ in. cards on which were typed (one to a card in upper case letters) either the stimuli of list 1 (for the RF condition) or those of list 2 (the IF condition). Each stimulus was presented 7 times. The packs were shuffled after each S's use. In addition, Ss were given a 7 page booklet containing 84 lines (12 to a page) in which to write the trigrams. Ss were instructed either to (a) spell each trigram to himself three times while writing it (the Spell condition) or to (b) pronounce each trigram to himself three times while writing it (the Pronounce condition). Thus, in combination with the two lists, there are four familiarization conditions: Pronounce Relevant Familiarization(PRF), Pronounce Irrelevant Familiarization (PIF), Spell Relevant Familiarization (SRF), and Spell Irrelevant Familiarization (SIF).

Association Test: In individual sessions which immediately followed the familiarization procedure, Ss were given an association test. The instructions (same for all groups) stressed that Ss should respond as soon as possible after seeing the trigrams. The stimuli (of list 1 ) were typed in upper case letters on 3 in. $x 5$ in. cards. Timings were taken to the nearest $.01 \mathrm{sec}$. from the exposure of a stimulus trigram to the onset of S's response. The stimuli were shuffled after each use. Five practice stimuli were used to acquaint $S s$ with the procedure.

Learning Task: In individual sessions immediately following the association test, Ss were required to learn (by serial anticipation) the 12 stimuli in list 1. The instructions required Ss to respond by spelling the trigrams. The list was presented by a Gerbrands memory drum set as follows: 3 sec. exposure per item, zero inter-item interval, and $6 \mathrm{sec}$. intertrial interval. The criterion of mastery was two successive perfect recitations. The dependent variable was the total number of errors to criterion.

\section{Results}

Associative Latency: The associative latencies of each S's 12 responses (to the stimuli of list 1) were summed. These data were then subjected to median tests. All $p$ values represent one-tailed tests.

$\mathrm{H}_{1}$ : A median test of the responses of the group $(\mathrm{N}=22)$ receiving pronouncing instructions produced a $\mathrm{X}^{2}=2.93$ which for $\mathrm{df}=1, \mathrm{p}<.05$ and it is concluded that the PRF group responded faster than the PIF group. The medians are $19.47 \mathrm{sec}$. and $31.89 \mathrm{sec}$. for the PRF and PIF groups respectively. Hypothesis $H_{1}$ is, therefore, confirmed. Familiarization with instructions to pronounce the materials has decreased these Ss' associative latency.

$\mathrm{H}_{2}$ : A median test of the responses of the group $(\mathrm{N}=22)$ receiving spelling instructions produced a $\mathrm{X}^{2}=.73$ which for $\mathrm{df}=1, .25>\mathrm{p}>.15$ and it is concluded that the medians of these two groups (SRF and SIF) are 
equal. The SRF median is $24.75 \mathrm{sec}$, and the SIF median is $30.21 \mathrm{sec}$. Hypothesis $\mathrm{H}_{2}$ is also confirmed. Instructions to spell the materials do not produce the same effect as instructions to pronounce.

$\mathrm{H}_{3}$ : The directionality of the difference between the PRF and SRF groups is such that the PRF group was faster (PRF median=19.47 sec. vs $24.75 \mathrm{sec}$. for the SRF group). However, a median test showed that the difference is not significant: $X^{2}=.73$ which for $d f=1$, $.25>\mathrm{p}>\mathbf{. 1 5}$. Hypothesis $\mathrm{H}_{3}$ is not confirmed.

The only other comparison which produced significance was that of the RF and IF groups: $X^{2}=3.27$ which for $\mathrm{df}=1, \mathrm{p}<.05$. The RF group, with a median of 23.16 sec., was the faster (IF median $=30.58$ seconds).

Learning: A median test of the learning data disclosed that the PRF group attained the proficiency criterion with significantly fewer errors than the PIF group: $x^{2}=6.60$ which for $d f=1, p<.01$. The median for the PRF group is 78 errors and for the PIF group is 132 errors.

There was no difference between the SRF and the SIF groups on this learning task $\left(X^{2}=0\right)$. As before, the difference between the PRF and SRF groups is not significant $\left(X^{2}=.73\right.$ which for $\left.d f=1, .25>p>.15\right)$ but in favor of the PRF group. The respective medians are 78 errors (PRF) and 112 errors (SRF).

As was found with the latency data, there was an overall RF group superiority: $X^{2}=3.27$ which for $\mathrm{df}=1, \mathrm{p}<.05$. The median RF performance was 84 errors and the median IF score was 134 errors.

In general, significant differences in the learning task are found where there are significant differences in $\mathrm{AL}$ ( $\mathrm{PRF}>\mathrm{PIF}$ and $\mathrm{RF}>\mathrm{IF}$ ).

\section{Discussion}

It is now established that a relatively small amount of familiarization (f) can decrease the latency of Ss' as sociations to CVC trigrams. This effect, furthermore, is neither list nor subject specific. One determinant of the effect (there may be others) is the $f$ instructions given S6. A significant decrease in AL has been found so far only with instructions to pronounce the materials.

It is also established that a relatively small amount of $f$ with instructions to pronounce the materials facilitates Ss' learning a list of CVC trigrams whereas the same amount of $f$ with instructions to spell the materials did not produce facilitation. Presumably, the SRF group was not superior to the SIF controls on the learning task either because of the small number of $f$ trials or because the learning task was not sufficiently difficult to allow whatever superiority that may exist to become evident (q.v, Riley \& Phillips).

It has been known for some time that $\mathrm{AL}$ is negatively correlated with ease of learning (Johnson, 1964) but the above data seem to extend our knowledge of the role played by AL by suggesting that the AL of verbal materials is functionally related to the ease with which they are learned.

With respect to the effect of $f$ on meaningfuiness $(\mathrm{mf})$, it has been stated (Gillooly, 1965) that, "... it is (now) more difficult to maintain that $\mathrm{mf}$ and familiarity are independent concepts (Noble, 1963) .... If the latency of the first associate to CVC trigrams is significantly reduced by $f$ training, presumably associative frequency is increased (by increasing the probability that an associate will be reported in a constant amount of time and/or by leaving Ss more of that constant amount of time in which to report additional associations). "Since that time, however, Schulz \& Thysell (1965) have reported findings which support and extend those of Riley and Phillips by showing that with dissyllables and several degrees of $f$, associative productivity (AP) remains unaffected (in 30 sec.) despite the fact that their instructions required Ss to pronounce the materials to themselves. These results require that speculation about AP cease. Although $f$ decreases AL, AP remains unaltered. Schulz and Thysell conclude "The results of the present study, when considered in conjunction with those obtained previously by Riley \& Phillips (1959), would seem to justify a firm conclusion that familiarization does not affect $\mathrm{m}$. Furthermore this conclusion now has considerable generality ....."

However, the general problem of deciding whether or not $f$ affects $\mathrm{mf}$ can only be resolved by choosing among the several definitions of $\mathrm{mf}$. If by $\mathrm{mf}$ one means the number of associations produced in a fixed amount of time (Noble's $\underline{m}$ used by Schulz and Thysell), then he is correct in stating that $m f$ is unaffected by $f$. However, if one defines the $\mathrm{mf}$ of stimuli as being related to the probability that an association will be reported in a fixed amount of time (the familiar Association Value invented by Glaze, 1928, and used by Archer, 1960, among others), then reducing $A L$ would seem to increase this index of $\mathrm{mf}$.

If one wishes to be most direct by asserting that the mf of stimuli is inversely related to the time required to respond with an association (the $\bar{M}$ scale is based on this definition; Gillooly, 1964), then the above reported results demonstrate that $f$ does increase the $\mathrm{mf}$ of $\mathrm{CVC}$ trigrams.

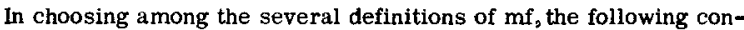
siderations seem important: (1) When science can riditself of a concept by choosing one instead of another definition, that concept is superfluous. Choosing an associative latency-related definition of $\mathrm{mf}$ is the parsimonious thing to do. (2) It seems desirable to use as an index of $\mathrm{mf}$ one which is sensitive to the maximum number of influences which affect the ease with which verbal material is learned. Noble's $\underline{m}$, by being insensitive to the effects of $f$, resulted in the mystique of familiarity. Defining $\mathrm{mf}$ in terms of AL eliminates this problem. Familiarization is seen as a technique for altering the $\mathrm{mf}$ of stimulinot as an independent dimension of verbal material.(3) Regarding mf as being dependent upon f procedures is credible. How else did verbal stimuli acquire whatever $\mathrm{mf}$ they possess if not through $f$ procedures (which more often than not involved use of pronunciation) or generalization from familiarized stimuli?

\section{References}

Archer, E. James. A re-evaluation of the meaningfulness of all possible CVC trigrams. Psychol. Monogr., 1960, 74, No. 10 (Whole No. 497).

Gillooly, William B. Meaningfulness as a function of the latency of association. Unpublished doctoral dissertation, The Johns Hopkins University, Baltimore, Maryland, 1964.

Gillooly, William B. The effect of familiarization on associative latency. Psychon. Sci., 1965, 3, 235-236.

Glaze, J. A. The association value of non-sense syllables. $J$. genet. Psychol., 1928, 35, 255-267.

Hilgard, E.R. Methods and procedures in the study of learning. In S. S. Stevens (Ed.), Handbook of experimental psychol., New York: Wiley \& Sons, 1951.

Johnson, R. C. Latency and association value as ptedictors of rate of verbal learning. J. verbal Learn. verbal Behav., 1964, 3, 77-78.

Noble, C. E. Meaningfulness and familiarity. In C. N. Cofer \& B. S. Musgrave (Eds.), Verbal behavior and learning. New York: McGraw-Hill, 1963.

Riley, D. A., \& Phillips, L. W. The effects of syllable familiarization on rote learning, association value, and reminiscence. J. exp. Psychol., 1959, 57, 372-379.

Schulz, R. W., \& Thysell, R. The effect of familiarization on meaningfulness. J. verbal Learn. verbal Behav., 1965, 4, 409 413 